PROCEEDINGS OF THE

AMERICAN MATHEMATICAL SOCIETY

Volume 130, Number 10, Pages 2837-2843

S 0002-9939(02)06399-2

Article electronically published on March 14, 2002

\title{
FORCING A BOOLEAN ALGEBRA WITH PREDESIGNED AUTOMORPHISM GROUP
}

\author{
TAPANI HYTTINEN AND SAHARON SHELAH
}

(Communicated by Carl G. Jockusch, Jr.)

\begin{abstract}
For suitable groups $G$ we will show that one can add a Boolean algebra $B$ by forcing in such a way that $A u t(B)$ is almost isomorphic to $G$. In particular, we will give a positive answer to the following question due to J. Roitman: Is $\aleph_{\omega}$ a possible number of automorphisms of a rich Boolean algebra?
\end{abstract}

In [Ro], J. Roitman asked, is $\aleph_{\omega}$ a possible number of automorphisms of a rich Boolean algebra? A Boolean algebra is rich if the number of automorphisms is greater than the size of the algebra and possible means consistent with ZFC (since GCH implies trivially that the answer is no). We will answer this question positively. For this we will give a method for adding a Boolean algebra by forcing in such a way that we have a lot of control on the automorphism group of the Boolean algebra. Notice that by $[\mathrm{Mo}$, Theorem 4.3, one cannot hope of giving a positive answer to Roitman's question from the assumption that $2^{\omega}>\aleph_{\omega}$.

Partially, our methods are similar to those in [Ro].

We say that a po-set $P$ is $\kappa$-Knaster if for all $p_{i} \in P, i<\kappa$, there is a set $Z \subseteq \kappa$ of power $\kappa$ such that for all $i, j \in Z, p_{i}$ and $p_{j}$ are compatible.

1. Theorem. Assume that $\kappa=\kappa^{<\kappa}$ is an infinite cardinal. Let $(I, \leq)$ be a partial order, $G_{t}, t \in I$, be groups of power $\leq \kappa$ and $\pi_{s, t}: G_{t} \rightarrow G_{s}, s<t \in I$, be homomorphisms such that

(a) I is $\kappa^{+}$-directed, every pair of elements of I has the least upper bound and for all $t \in I,|\{s \in I \mid s \leq t\}| \leq \kappa$,

(b) for all $r<s<t \in I, \pi_{r, t}=\pi_{r, s} \circ \pi_{s, t}$.

Then there is a $\kappa$-closed, $\kappa^{+}$-Knaster po-set $P$ such that in $V^{P}$ the following holds: There is a Boolean algebra $B$ such that

(i) $B$ is atomic with $|I|+\kappa$ atoms,

(ii) $|B|=\kappa^{+}+|I|$,

(iii) if $G^{*}$ is the inverse limit of $\left(G_{t}, \pi_{s, t} \mid s<t \in I\right)$, then $G^{*}$ can be embedded into $\operatorname{Aut}(B),|A u t(B)| \leq \kappa^{+} \cdot|I| \cdot\left|G^{*}\right|$ and if for all $t \in I, G_{t}$ is the one element group, then $\mid$ Aut $(B)|=\kappa \cdot| I \mid$.

Received by the editors January 31, 2001 and, in revised form, May 14, 2001.

2000 Mathematics Subject Classification. Primary 06E05; Secondary 03E35.

The first author was partially supported by the Academy of Finland, grant 40734, and the Mittag-Leffler Institute.

The research of the second author was supported by the Israel Science Foundation founded by the Israel Academy of Sciences and Humanities. Publ. 756. 
Note: We will show that $\operatorname{Aut}(B)$ is not very far from $G^{*}$. In particular, we will show that for all $f \in A u t(B)$ there is $g \in G^{*}$ such that excluding a small error, $f$ is the same as the image of $g$ under the embedding.

Proof. For $t \in I$, let $X_{t}=\{t\} \times G_{t} \times \kappa, X_{\leq t}=\bigcup_{s<t} X_{s}$ and $X=\bigcup_{t \in I} X_{t}$. We define $P$ to be the set of all tuples $p=(U, T, V, Y)=\left(U^{p}, T^{p}, V^{p}, Y^{p}\right)$ such that

(1) $U \subseteq X$ is of power $<\kappa$,

(2) $T \subseteq I$ is of power $<\kappa$,

(3) $V=\left(v_{t} \mid t \in T\right)=\left(v_{t}^{p} \mid t \in T\right)$, where each $v_{t} \subseteq \kappa^{+}$is of power $<\kappa$,

(4) $Y=\left(y_{t, \alpha} \mid t \in T, \alpha \in v_{t}\right)=\left(y_{t, \alpha}^{p} \mid t \in T, \alpha \in v_{t}\right)$, where $y_{t, \alpha} \subseteq U \cap X_{\leq t}$.

We order $P$ so that $p \leq q(q$ is stronger than $p)$ if

(5) $U^{p} \subseteq U^{q}$

(6) $T^{p} \subseteq T^{q}$

(7) $v_{t}^{p} \subseteq v_{t}^{q}$ for $t \in T^{p}$,

(8) $y_{t, \alpha}^{p}=y_{t, \alpha}^{q} \cap U^{p}$ for $t \in T^{p}$ and $\alpha \in v_{t}^{p}$.

Clearly $P$ is $\kappa$-closed and $\kappa^{<\kappa}=\kappa$ implies by the usual $\Delta$-lemma argument that $P$ is $\kappa^{+}$-Knaster.

Let $H$ be $P$-generic over $V$. We let $Y_{t, \alpha}=\bigcup\left\{y_{t, \alpha}^{p} \mid p \in H\right\}$. Below, we will not distinguish these and other objects from their names; it will be clear from the context which we mean.

Let $G^{*}$ be as in the theorem, i.e. $G^{*}$ consists of all $g: I \rightarrow \bigcup_{t \in I} G_{t}$ such that for all $s<t \in I, \pi_{s, t}(g(t))=g(s)$. If $g \in G^{*}$ and $x=(t, z, \alpha) \in X_{t}$, then we also write $g(x)$ for $(t, g(t) z, \alpha)$ and define $Y_{t, \alpha, g}=\left\{g(x) \mid x \in Y_{t, \alpha}\right\}$.

By a Boolean term $\delta\left(Y_{0}, \ldots, Y_{n-1}\right)$ taken inside $Y$ we mean a term of the form $\bigcap_{i<n} Z_{i}$, where each $Z_{i}$ is either $Y \cap Y_{i}$ or $Y-Y_{i}$. If $Z_{i}=Y \cap Y_{i}$ we say that $Y_{i}$ appears in $\delta$ positively. A general Boolean term is a finite union of Boolean terms.

1.1. Claim. Let $\delta\left(Y_{0}, \ldots, Y_{n-1}\right)$ be a Boolean term taken inside $X_{\leq t}, t \in I$, and for $i<n, t_{i} \in I, \alpha_{i}<\kappa^{+}$and $g_{i} \in G^{*}$. Assume that there is $t^{\prime} \in I$ such that

(a) $t^{\prime} \leq t$

(b) if $Y_{i}$ appears in $\delta$ positively, then $t^{\prime} \leq t_{i}$,

(c) if $Y_{i}$ appears in $\delta$ positively and $Y_{j}$ does not appear positively in $\delta$, then $\left(t_{i}, \alpha_{i}, g_{i}\left(t^{\prime}\right)\right) \neq\left(t_{j}, \alpha_{j}, g_{j}\left(t^{\prime}\right)\right)$.

Then $\delta\left(Y_{t_{0}, \alpha_{0}, g_{0}}, \ldots, Y_{t_{n-1}, \alpha_{n-1}, g_{n-1}}\right) \cap X_{\leq t^{\prime}}$ has power $\kappa$. On the other hand, if such $a t^{\prime}$ does not exist, then $\delta\left(Y_{t_{0}, \alpha_{0}, g_{0}}, \ldots, Y_{t_{n-1}, \alpha_{n-1}, g_{n-1}}\right)=\emptyset$.

Proof. An easy density argument using the fact that the actions of two distinct elements of a group differ on each element of the group proves Claim 1.1.

Now we can define the Boolean algebra $B$. We let $B$ be the subalgebra of $\mathcal{P}(X)$ generated by $[X]^{<\kappa} \cup\left\{Y_{t, \alpha, g} \mid t \in I, \alpha<\kappa^{+}, g \in G^{*}\right\}$, where $[X]^{<\kappa}$ is the set of all subsets of $X$ of power $<\kappa$.

1.2. Claim. Items (i) and (ii) from Theorem 1 hold and $G^{*}$ can be embedded into $\operatorname{Aut}(B)$.

Proof. (i) is clear. For (ii), notice that $Y_{t, \alpha, g}$ is determined by $Y_{t, \alpha}$ and $g(t)$. Finally, it is easy to check that the function which takes $g \in G^{*}$ to $\bar{g}$ is the required embedding of $G^{*}$ into $A u t(B)$, where $\bar{g}$ is such that it maps each $A \in B$ to $\{g(z) \mid z \in$ $A\}$. This proves Claim 1.2. 
So we are "left" to prove the second half of (iii) from Theorem 1: For all $0<m<$ $\omega$ and $t \in I$, let $\delta_{i}^{t, m}\left(Y_{0}, \ldots, Y_{m-1}\right), i<2^{m}$, list all Boolean terms in $m$ variables taken inside $X_{\leq t}$ so that $\delta_{0}^{t, m}\left(Y_{0}, \ldots, Y_{m-1}\right)=\bigcap_{k<m}\left(X_{\leq t}-Y_{k}\right)$ and for $m=0$, we let $\delta_{0}^{t, m}=X_{\leq t}$.

Let $h \in A u t(B)$. By the definition of $B, h$ is determined by $h \uparrow\{\{x\} \mid x \in X\}$. In order to simplify the notation, for $x \in X$, we write $h(x)$ for the unique $z \in X$ such that $h(\{x\})=\{z\}$. We will frequently consider such an $h$ as if it is a permutation of $X$.

1.3. Claim. In $V^{P}$, if $h \in A u t(B)$ and $t \in I$, then there are $u \subseteq X_{\leq t}, m<\omega$, for $i<m, t_{i} \in I, \alpha_{i}<\kappa^{+}$and $g_{i} \in G^{*}$ and for $j<2^{m}, f_{j} \in G^{*}$ such that

(i) $|u|<\kappa$,

(ii) either for all $j<2^{m}$ and $x \in \delta_{j}^{t, m}\left(Y_{t_{0}, \alpha_{0}, g_{0}}, \ldots, Y_{t_{m-1}, \alpha_{m-1}, g_{m-1}}\right)-u, h(x)=$ $f_{j}(x)$ or for all $j<2^{m}$ and $x \in \delta_{j}^{t, m}\left(Y_{t_{0}, \alpha_{0}, g_{0}}, \ldots, Y_{t_{m-1}, \alpha_{m-1}, g_{m-1}}\right)-u, h^{-1}(x)=$ $f_{j}(x)$.

Proof. For a contradiction, assume that $p$ forces that $h$ and $t$ are a counterexample. For all $x \in X_{\leq t}$, let $\left(p_{x, i}\right)_{i<\kappa}$ be a maximal antichain such that for all $i<\kappa$, $p_{x, i} \geq p$ or $p_{x, i}$ is incompatible with $p$ and $p_{x, i}$ decides both $h(x)$ and $h^{-1}(x)$. Choose $\alpha^{*}<\kappa^{+}$so that

$$
\alpha^{*} \notin \bigcup\left\{v_{t}^{p_{x, i}} \mid x \in X_{\leq t}, i<\kappa\right\} .
$$

Choose $q \geq p$ so that it decides both $h\left(Y_{t, \alpha^{*}}\right)$ and $h^{-1}\left(Y_{t, \alpha^{*}}\right)$, i.e. it forces $h\left(Y_{t, \alpha^{*}}\right)$

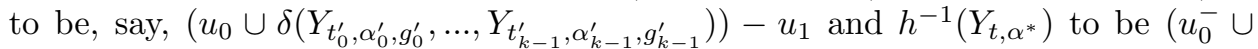
$\left.\delta^{-}\left(Y_{t_{0}^{\prime}, \alpha_{0}^{\prime}, g_{0}^{\prime}}, \ldots, Y_{t_{k-1}^{\prime}, \alpha_{k-1}^{\prime}, g_{k-1}^{\prime}}\right)\right)-u_{1}^{-}$, where $u_{0}, u_{1}, u_{1}^{-}, u_{2}^{-} \in[X]^{<\kappa}$ and $\delta$ and $\delta^{-}$ are general Boolean terms taken inside $X$. (Notice that $Y_{t, \alpha^{*}}=Y_{t, \alpha^{*}, 1}$.) In particular, $q$ is chosen so that it decides values for $g_{i}^{\prime}\left(t_{i}^{\prime}\right)$ and $g_{i}^{\prime}(t), i<k$. Clearly we may assume that ( $q$ forces that) in $\left(\left(t_{0}^{\prime}, \alpha_{0}^{\prime}, g_{0}^{\prime}\left(t_{0}^{\prime}\right)\right), \ldots,\left(t_{k-1}^{\prime}, \alpha_{k-1}^{\prime}, g_{k-1}^{\prime}\left(t_{k-1}^{\prime}\right)\right)\right)$ there is no repetition, and for some $k^{*} \leq k,\left\{i<k \mid\left(t_{i}^{\prime}, \alpha_{i}^{\prime}\right)=\left(t, \alpha^{*}\right)\right\}=k^{*}$. We write $\bar{Y}_{<k^{*}}$

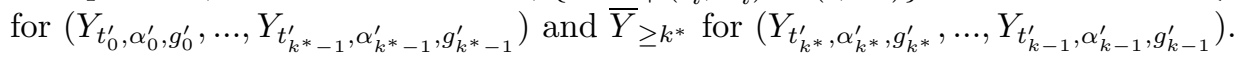

If we can find $x \in X_{\leq t}-U^{q}$ and $q^{\prime} \geq q$ such that $q^{\prime}$ forces that for all $i<$ $k^{*}, g_{i}^{\prime}(x) \neq h(x)$ and that $h(x) \notin u_{0} \cup u_{1}$, we get an easy contradiction. The contradiction is obtained the same way as we obtain a contradiction after the proof of Subclaim 1.3.1 (we can find $q^{\prime} \geq q$ so that it forces $h(x) \in h\left(Y_{t, \alpha^{*}}\right) \leftrightarrow h(x) \notin$ $\delta(\bar{Y}))$. Similarly, if we can find $x \in X_{\leq t}-U^{q}$ and $q^{\prime} \geq q$ such that $q^{\prime}$ forces that for all $i<k^{*}, g_{i}^{\prime}(x) \neq h^{-1}(x)$ and that $h^{-1}(x) \notin u_{0}^{-} \cup u_{1}^{-}$, we get an easy contradiction. So we assume the following:

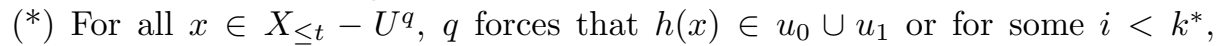
$h(x)=g_{i}^{\prime}(x)$ and that $h^{-1}(x) \in u_{0}^{-} \cup u_{1}^{-}$or for some $i<k^{*}, h^{-1}(x)=g_{i}^{\prime}(x)$.

Notice that $\left(^{*}\right)$ implies that for all $t^{\prime} \leq t, q$ forces that excluding a small error, $h$ is a permutation of $X_{t^{\prime}}$.

By $\left(^{*}\right)$ above and the choice of $p, h$ and $t$, and by making $q$ stronger if necessary, we may assume that there are Boolean terms $\delta^{\prime}$ and $\delta^{\prime \prime}$ taken inside $X_{\leq t}$ such that

$(\alpha) q$ forces that for all $g \in G^{*}$ there are $\kappa$ many $x \in X_{\leq t}$ such that $g(x) \neq h(x) \in$ $\delta^{\prime}\left(\bar{Y}_{<k^{*}}\right) \cap \delta^{\prime \prime}\left(\bar{Y}_{\geq k^{*}}\right)$,

( $\beta) q$ forces that $\delta^{\prime}\left(\bar{Y}_{<k^{*}}\right) \cap \delta^{\prime \prime}\left(\bar{Y}_{\geq k^{*}}\right) \neq \emptyset$,

$(\gamma)$ every condition forces that $\delta^{\prime}\left(\bar{Y}_{<k^{*}}\right) \cap \delta^{\prime \prime}\left(\bar{Y}_{\leq k^{*}}\right) \subseteq \delta(\bar{Y})$. 
Instead of item $(\gamma)$, we may have $\delta^{\prime}\left(\bar{Y}_{<k^{*}}\right) \cap \delta^{\prime \prime}\left(\bar{Y}_{\leq k^{*}}\right) \subseteq X_{\leq t}-\delta(\bar{Y})$. This is a symmetric case and we skip it.

1.3.1. Subclaim. There are $x_{1}, x_{2} \in X_{\leq t}, d_{1}, d_{2} \in X_{\leq t}, q^{*} \geq q$ and non-empty $W_{1}, W_{2} \subseteq k^{*}$ such that

(1) $W_{1} \cap W_{2}=\emptyset$,

(2) $q^{*}$ forces that for all $n \in\{1,2\}, h\left(x_{n}\right)=d_{n}$,

(3) $q^{*}$ forces that for all $n \in\{1,2\}, d_{n} \in \delta^{\prime \prime}\left(\bar{Y}_{\geq k^{*}}\right)-\left(u_{0} \cup u_{1}\right)$,

(4) $q^{*}$ forces that for all $n \in\{1,2\}$ and $i<k^{*}, d_{n}=g_{i}^{\prime}\left(x_{n}\right)$ iff $i \in W_{n}$,

(5) if $d_{1} \in X_{v_{1}}$ and $d_{2} \in X_{v_{2}}$, then $v_{1} \leq v_{2}$,

(6) for all $n \in\{1,2\}$ and $i<k^{*},\left(g_{i}^{\prime}\right)^{-1}\left(d_{n}\right) \notin U^{q}$ and $\left\{\left(g_{i}^{\prime}\right)^{-1}\left(d_{1}\right) \mid i<k^{*}\right\} \cap$ $\left\{\left(g_{i}^{\prime}\right)^{-1}\left(d_{2}\right) \mid i<k^{*}\right\}=\emptyset$.

Proof. Let $I^{\prime} \subseteq I$ be the set of those $t^{\prime} \leq t$ such that $X_{t^{\prime}} \cap \delta^{\prime \prime}\left(\bar{Y}_{\leq k^{*}}\right) \neq \emptyset$. Notice that by Lemma 1.1, q decides $I^{\prime}$ and $I^{\prime}$ is closed under the least upper bounds. By $(\beta)$ above, $I^{\prime} \neq \emptyset$. For $t^{\prime} \in I^{\prime}$, we write $I_{\geq t^{\prime}}^{\prime}$ for $\left\{v \in I^{\prime} \mid v \geq t^{\prime}\right\}$. Then for all $t^{*} \in I^{\prime}$ there are $W^{*} \subseteq k^{*}$ and $q^{\prime} \geq q$, such that $q^{\prime}$ forces that excluding $<\kappa$ many $x$ if $h(x) \in \delta^{\prime \prime}\left(\bar{Y}_{\geq k^{*}}\right)-\left(u_{0} \cup u_{1}\right)$ and $x \in X_{v}$ for some $v \in I_{\geq t^{\prime}}^{\prime}$, then $h(x)=g_{i}^{\prime}(x)$ for some $i \in W^{*}$. We choose these so that $\left|W^{*}\right|$ is minimal. There are two cases:

(I) $\left|W^{*}\right|>1$ : Now it is easy to choose $v_{1}, v_{2} \in I_{\geq t^{*}}^{\prime}, x_{1} \in X_{v_{1}}, x_{2} \in X_{v_{2}}$, $d_{1}, d_{2} \in X_{\leq t}, i \in W^{*}$ and $q^{*} \geq q^{\prime}$ so that $v_{2} \geq v_{1}$, for $n \in\{1,2\}, q^{*}$ forces that $h\left(x_{n}\right)=d_{n}, h\left(x_{n}\right) \in \delta^{\prime \prime}\left(\bar{Y}_{\geq k^{*}}\right)-\left(u_{0} \cup u_{1}\right), h\left(x_{1}\right) \neq g_{i}^{\prime}\left(x_{1}\right)$ and $h\left(x_{2}\right)=g_{i}^{\prime}\left(x_{2}\right)$. Then we let $W_{1}=\left\{j<k^{*} \mid g_{j}^{\prime}\left(x_{1}\right)=d_{1}\right\}$ and $W_{2}=\left\{j<k^{*} \mid g_{j}^{\prime}\left(x_{2}\right)=d_{2}\right\}$. By the choice of $W^{*}$, these can be chosen so that in addition (6) holds. These are as wanted $\left(v_{2} \geq v_{1}\right.$ and $h\left(x_{1}\right) \neq g_{i}^{\prime}\left(x_{1}\right)$ imply $\left.(1)\right)$.

(II) $\left|W^{*}\right|=1$ : Let $i^{*}$ be the only element of $W^{*}$. By $(\alpha)$ above, we can find $q^{\prime \prime} \geq q^{\prime}, x_{1} \in X_{\leq t}-U^{q}$ and $d_{1} \in X_{\leq t}$, so that $q^{\prime \prime}$ forces that $h\left(x_{1}\right)=d_{1}, h\left(x_{1}\right) \in$ $\delta^{\prime \prime}\left(\bar{Y}_{\geq k^{*}}\right)-\left(u_{0} \cup u_{1}\right)$ and $h\left(x_{1}\right) \neq g_{i^{*}}^{\prime}\left(x_{1}\right)$. Let $v_{1} \in I$ be such that $x_{1} \in X_{v_{1}}$ and let $v_{2}$ be the least upper bound of $v_{1}$ and $t^{*}$. By the assumption $\left(^{*}\right)$ above, $d_{1} \in X_{v_{1}}$ and so $v_{2} \in I_{\geq t^{*}}^{\prime}$. Then by Claim 1.1 and the assumption that $\left|W^{*}\right|=1$, there are $x_{2} \in X_{v_{2}}, d_{2} \in X_{\leq t}$ and $q^{*} \geq q^{\prime \prime}$ such that $q^{*}$ forces that $h\left(x_{2}\right)=d_{2}=g_{i^{*}}^{\prime}\left(x_{2}\right)$ and $h\left(x_{2}\right) \in \delta^{\prime \prime}\left(\bar{Y}_{\geq k^{*}}\right)-\left(u_{0} \cup u_{1}\right)$. Then we can let $W_{1}=\left\{j<k^{*} \mid g_{j}^{\prime}\left(x_{1}\right)=d_{1}\right\}$ and $W_{2}=\left\{j<k^{*} \mid g_{j}^{\prime}\left(x_{2}\right)=d_{2}\right\}$. By $(\alpha)$ and the choice of $W^{*}$, these can be chosen so that in addition (6) holds. These are as wanted and proves Subclaim 1.3.1.

By the choice of the conditions $p_{x, i}$, we can find $r^{\prime} \geq q^{*}$ and $i, i^{\prime}<\kappa$ so that $p_{x_{1}, i}, p_{x_{2}, i^{\prime}} \leq r^{\prime}$.

We define a condition $r$ as follows:

(a) $U^{r}=U^{r^{\prime}} \cup\left\{\left(g_{i}^{\prime}\right)^{-1}\left(d_{n}\right) \mid i<k, n \in\{1,2\}\right\}$,

(b) $T^{r}=T^{r^{\prime}} \cup\{t\}$,

(c) $v_{s}^{r}=v_{s}^{r^{\prime}} \cup\left\{\alpha^{*}\right\}$,

(d) if $(s, \alpha) \neq\left(t, \alpha^{*}\right)$, then $y_{s, \alpha}^{r}=y_{s, \alpha}^{r^{\prime}}$,

(e) $y_{t, \alpha^{*}}^{r}=y_{t, a^{*}}^{q} \cup\left\{\left(g_{i}^{\prime}\right)^{-1}\left(d_{n}\right) \mid i \in W_{1}, n \in\{1,2\}\right\}$.

Then $r \geq q, p_{x_{1}, i}, p_{x_{2}, i^{\prime}}$; in particular $r$ forces that $h\left(x_{1}\right)=d_{1}$ and $h\left(x_{2}\right)=d_{2}$. Let $\delta^{*}\left(Y_{0}, \ldots, Y_{k^{*}-1}\right)$ be a boolean term taken inside $X_{\leq t}$ so that $Y_{i}$ appears in $\delta^{*}$ positively iff $i \in W_{1}$. Then using Subclaim 1.3.1, it is easy to check that $r$ forces that $d_{1} \in \delta^{*}\left(\bar{Y}_{<k^{*}}\right) \cap \delta^{\prime \prime}\left(\bar{Y}_{\geq k^{*}}\right)-\left(u_{0} \cup u_{1}\right)$ and $d_{1} \in h\left(Y_{t, \alpha^{*}}\right)$. Since $r \geq q$, this implies that $r$ forces that $\delta^{*}\left(\bar{Y}_{<k^{*}}\right) \cap \delta^{\prime \prime}\left(\bar{Y}_{\geq k^{*}}\right)-u_{1} \subseteq \delta(\bar{Y})-u_{1} \subseteq h\left(Y_{t, \alpha^{*}}\right)$. On 
the other hand, using Subclaim 1.3.1 (1), (4) and (5), $r$ forces that $d_{2} \in \delta^{*}\left(\bar{Y}_{<k^{*}}\right) \cap$ $\delta^{\prime \prime}\left(\bar{Y}_{\geq k^{*}}\right)-\left(u_{0} \cup u_{1}\right)$ and $d_{2} \notin h\left(Y_{t, \alpha^{*}}\right)$, a contradiction which proves Claim 1.3.

From now on we work in $V^{P}$.

1.4. Claim. Suppose $h \in \operatorname{Aut}(B)$. There are $h^{*}, s^{*} \in I u^{*} \subseteq X_{\leq s^{*}}, m^{*}<\omega$, for $i<m^{*}, t_{i}^{s^{*}} \in I, \alpha_{i}^{s^{*}}<\kappa^{+}, g_{i}^{s^{*}} \in G^{*}$, for $0<j<2^{m^{*}}, f_{j}^{s^{*}} \in G^{*}$ and $f^{*} \in G^{*}$ such that if we write $\bar{Y}=\left(Y_{t_{0}^{* *}, \alpha_{0}^{s^{*}}, g_{0}^{s^{*}}}, \ldots, Y_{t_{m^{*}-1}^{s^{*}}, \alpha_{m^{*}-1}^{s^{*}}, g_{m^{*}-1}^{s^{*}}}\right)$, then the following hold:

(i) $h^{*}=h$ or $h^{*}=h^{-1}$,

(ii) for all $0<j<2^{m^{*}}$ and $x \in \delta_{j}^{s^{*}, m^{*}}(\bar{Y})-u^{*}, h^{*}(x)=f_{j}^{s^{*}}(x)$,

(iii) for all $x \in\left(X-X_{\leq s^{*}}\right) \cup\left(\delta_{0}^{s^{*}, m^{*}}(\bar{Y})-u^{*}\right), h^{*}(x)=f^{*}(x)$,

(iv) for all $i<m^{*}, t_{i}^{s^{*}} \leq s^{*}$,

(v) $\left|u^{*}\right|<\kappa$.

Proof. For every $s \in I$, choose $u^{s}, m^{s}, t_{i}^{s}, \alpha_{i}^{s}, g_{i}^{s}$ and $f_{j}^{s}, i<m^{s}$ and $j<2^{m^{s}}$, as in Claim 1.3. We let $I^{*}$ to be the set of all $s \in I$ such that for all $j<2^{m^{s}}$

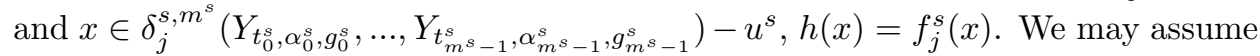
that $I^{*}$ is unbounded in $I$ (the case when $I-I^{*}$ is unbounded is similar) and we let $h^{*}=h$. We may also assume that these are chosen so that in addition $m^{s}$ and $u^{s}$ are minimal (in this order).

We write $E(s), s \in I^{*}$, for the set of all $(t, \alpha)$ such that for some $i<m^{s}, t=t_{i}^{s}$ and $\alpha=\alpha_{i}^{s}$.

1.4.1. Subclaim. If $r, s \in I^{*}$ and $r<s$, then

(a) $E(r) \subseteq E(s)$,

(b) for all $j<2^{m^{r}}, k<2^{m^{s}}$ and $t \leq r$, if

$$
\begin{aligned}
& \delta_{j}^{r, m^{r}}\left(Y_{t_{0}^{r}, \alpha_{0}^{r}, g_{0}^{r}, \ldots, Y_{t^{r}-1}^{r}, \alpha_{m}^{r} r_{1}, g_{m}^{r}{ }_{-1}}\right) \cap \\
& \quad \cap \delta_{k}^{s, m^{s}}\left(Y_{t_{0}^{s}, \alpha_{0}^{s}, g_{0}^{s}}, \ldots, Y_{t_{m}^{s} s_{-1}, \alpha_{m}^{s} s_{-1}, g_{m}^{s}{ }_{-1}}\right) \cap X_{t} \neq \emptyset,
\end{aligned}
$$

then $f_{j}^{r}(t)=f_{k}^{s}(t)$.

Proof. We will prove (a), (b) is immediate by Lemma 1.1. For a contradiction, assume that

(1) $\left(t_{0}^{r}, \alpha_{0}^{r}\right) \notin E(s)$.

We will write $\bar{Y}^{r}=\left(Y_{t_{0}^{r}, \alpha_{0}^{r}, g_{0}^{r}, \ldots, Y_{t^{r}-1}^{r}, \alpha_{m^{r}-1}^{r}, g_{m^{r}-1}^{r}}\right)$ and $\bar{Y}_{*}^{r}=\left(Y_{t_{1}^{r}, \alpha_{1}^{r}, g_{1}^{r}}, \ldots\right.$, $\left.Y_{t_{m^{r}-1}^{r}, \alpha_{m^{r}-1}^{r}, g_{m^{r}-1}^{r}}\right)$ and similarly for $s$ instead of $r$.

We will show that we can find $a, b<2^{m^{r}}, t \leq r$ and a Boolean term $\delta$ taken inside $X_{\leq r}$ such that

(2) $f_{a}^{r}(t) \neq f_{b}^{r}(t)$

(3) $\delta_{a}^{r, m^{r}}\left(\bar{Y}^{r}\right)=\left(X_{\leq r} \cap Y_{t_{0}^{r}, \alpha_{0}^{r}, g_{0}^{r}}\right) \cap \delta\left(\bar{Y}_{*}^{r}\right)$ and $X_{t} \cap \delta_{a}^{r, m^{r}}\left(\bar{Y}^{r}\right) \neq \emptyset$,

(4) $\delta_{b}^{r, m^{r}}\left(\bar{Y}^{r}\right)=\left(X_{\leq r}-Y_{t_{0}^{r}, \alpha_{0}^{r}, g_{0}^{r}}\right) \cap \delta\left(\bar{Y}_{*}^{r}\right)$ and $X_{t} \cap \delta_{b}^{r, m^{r}}\left(\bar{Y}^{r}\right) \neq \emptyset$.

If not, then for all $i<2^{m^{r}-1}$, we define $f_{i} \in G^{*}$ so that for all $x \in \delta_{i}^{r, m^{r}-1}\left(Y_{*}^{r}\right)-u^{r}$, $h(x)=f_{i}(x)$. This contradicts the minimality of $m^{r}$.

Let $J$ be the set of all $t \leq t_{0}^{r}$ such that $X_{t} \cap \delta_{i}^{r, m^{r}-1}\left(\bar{Y}_{*}^{r}\right) \neq \emptyset$. Notice that by Lemma 1.1, $J$ is closed under the least upper bounds. Let $c, d<2^{m^{r}}$ be such that $\delta_{c}^{r, m^{r}}\left(\bar{Y}^{r}\right)=\left(X_{\leq r} \cap Y_{t_{0}^{r}, \alpha_{0}^{r}, g_{0}^{r}}\right) \cap \delta_{i}^{r, m^{r}-1}\left(\bar{Y}_{*}^{r}\right)$ and $\delta_{d}^{r, m^{r}}\left(\bar{Y}^{r}\right)=\left(X_{\leq r}-Y_{t_{0}^{r}, \alpha_{0}^{r}, g_{0}^{r}}\right) \cap$ $\delta_{i}^{r, m^{r}-1}\left(\bar{Y}_{*}^{r}\right)$. Let $J_{c}$ be the set of all $t \in J$ such that $X_{t} \cap \delta_{c}^{r, m^{r}}\left(\bar{Y}^{r}\right) \neq \emptyset$. $J_{d}$ is 
defined similarly. Then either $J_{c}$ or $J_{d}$ is cofinal in $J$. If $J_{d}$ is cofinal, then we let $f_{i}=f_{d}^{r}$ and otherwise we let $f_{i}=f_{c}^{r}$.

We show that $f_{i}$ is as wanted. We may assume that $J_{d}$ is not cofinal; the other case is easy. Then since $J$ is closed under the least upper bounds, $J-J_{d}$ is cofinal. By Lemma 1.1, this means that there is $0<k<m^{r}$ such that for all $t \in J-J_{d}$, $\left(t_{k}^{r}, \alpha_{k}^{r}, g_{k}^{r}(t)\right)=\left(t_{0}^{r}, \alpha_{0}^{r}, g_{0}^{r}(t)\right)$ and $Y_{t_{k}^{r}, \alpha_{k}^{r}, g_{k}^{r}}$ appears positively in $\delta_{i}^{r, m^{r}-1}\left(\bar{Y}_{*}^{r}\right)$. But then $\delta_{i}^{r, m^{r}-1}\left(\bar{Y}_{*}^{r}\right) \subseteq Y_{t_{0}^{r}, \alpha_{0}^{r}, g_{0}^{r}}$ and so $f_{i}$ is as wanted.

We have shown the existence of $a, b, t$ and $\delta$.

By Claim 1.1 and the fact that $s \geq r$, there is $c<2^{m^{s}}$ such that $\delta_{c}^{s, m^{s}}\left(\bar{Y}^{s}\right) \cap$ $\delta_{a}^{r, m^{r}}\left(\bar{Y}^{r}\right) \cap X_{t} \not \subseteq u^{s}$. But then, by (1) above and Claim 1.1, $\delta_{c}^{s, m^{s}}\left(\bar{Y}^{s}\right) \cap \delta_{b}^{r, m^{r}}\left(\bar{Y}^{r}\right) \cap$ $X_{\leq t} \not u^{s}$. This contradicts (2) and proves Subclaim 1.4.1.

Since $I$ is $\kappa^{+}$-directed, $I^{*}$ is also $\kappa^{+}$-directed and so Subclaim 1.4.1 implies that there is $s^{*}$ such that $E\left(s^{*}\right)$ is maximal and for all $i<m^{*}=m^{s^{*}}, t_{i}^{s^{*}} \leq s^{*}$. By the choice of $u^{s}, s \in I^{*}$, it is easy to see that if $r, s \in I^{*}$ and $s^{*} \leq r \leq s$, then $u^{r} \subseteq u^{s}$. So $s^{*}$ can be chosen so that, in addition, for all $s^{*} \leq r \in I^{*}, u^{r}=u^{s^{*}}=u^{*}$. By Claim 1.1 and Subclaim 1.4.1, there is $f^{*} \in G^{*}$ such that for all $s \in I^{*}, f_{0}^{s}(s)=f^{*}(s)$. These are as wanted and completes the the proof of Claim 1.4.

Now it follows immediately from Claim 1.4 that $|A u t(B)| \leq \kappa^{+} \cdot|I| \cdot\left|G^{*}\right|$ and if for all $t \in I, G_{t}$ is the one element group, then $|A u t(B)| \leq \kappa \cdot|I|$. Finally, for $|A u t(B)| \geq \kappa \cdot|I|$, notice that every permutation of $X$ which is the identity on every element except on $<\kappa$ many, can be lifted to an automorphism of $B$.

2. Remark. Notice that in the proof of Theorem 1 one can add $\lambda>\kappa^{+}$subsets for the sets $X_{\leq t}$ making the Boolean algebra larger, and the proof still works giving $\left|G^{*}\right| \leq|\operatorname{Aut}(B)| \leq \lambda \cdot|I| \cdot\left|G^{*}\right|$.

Our aim was to use Theorem 1 to solve questions on the number of automorphisms of Boolean algebras. We start by first proving a result from [Ro].

3. Conclusion $([\mathrm{Ro}])$. Assume that $\kappa^{<\kappa}=\kappa$. Then there is a $\kappa$-closed, $\kappa^{+}$Knaster po-set $P$ of power $\kappa^{+}$such that in $V^{P}$ there is an atomic Boolean algebra $B$ of power $\kappa^{+}$with $|A u t(B)|=\kappa$.

Proof. Just choose $I=\{0\}$ and $G_{0}$ to be the one element group and apply Theorem 1 (with these choices of $I$ and $G_{0}$, our proof is essentially the same as the related proof in [Ro]).

We say that $T$ is a $\kappa$-tree if it is a tree of power $\kappa$ and of height $\kappa$. We write $T_{\alpha}$ for $\{t \in T \mid \operatorname{otp}(\{s \in T \mid s<t\})=\alpha\}$ and say that a $\kappa$-tree $T$ is narrow if for all $\alpha<\kappa,\left|T_{\alpha}\right|<\kappa$. We say that $b$ is a branch in a tree $T$ if $b$ is a linearly ordered downwards closed subtree of $T$ and the height of $b$ is the same as that of $T$.

4. Corollary. Assume that $\kappa^{<\kappa}=\kappa$ and that there is a narrow $\kappa^{+}$-tree with $\lambda>0$ branches. Then there is a $\kappa$-closed, $\kappa^{+}$-Knaster po-set $P$ of power $\kappa^{+}$with the following property: In $V^{P}$ there is an atomic Boolean algebra $B$ of power $\kappa^{+}$such that $\lambda \leq|A u t(B)| \leq \kappa^{+} \cdot \lambda$.

Proof. We apply Theorem 1: Clearly we may assume that every element of $T$ belongs to some branch. Then we let $I=\kappa^{+}$and for each $\alpha \in I$, let $G_{\alpha}$ be the free group generated by $T_{\alpha}$. Finally, let $\pi_{\alpha, \beta}$ be the natural projection along the branches of $T$. Then by Theorem 1, it is enough to show the following: 
4.1. Claim. The cardinality of the inverse limit of the system is $\omega \cdot \lambda\left(\right.$ in $\left.V^{P}\right)$.

Proof. It is enough to show that the forcing does not add branches to $T$ (since $c f\left(\kappa^{+}\right)>\omega$, the inverse limit is the free group generated by the "branches" of $T)$. For a contradiction assume that $p$ forces that $b$ is a new branch in $T$. For all $\alpha<\kappa^{+}$, choose $p_{\alpha} \geq p$ and $t_{\alpha} \in T_{\alpha}$ so that $p_{\alpha}$ forces that $t_{\alpha} \in b$. Since $P$ is $\kappa^{+}$-Knaster, there is $Z \subseteq \kappa^{+}$of power $\kappa^{+}$such that for all $\alpha, \beta \in Z, p_{\alpha}$ and $p_{\beta}$ are compatible (and so if $\alpha<\beta$, then $t_{\alpha}<t_{\beta}$ ). Then there are no $\alpha \in Z$ so that $p_{\alpha}$ forces that there are $\kappa^{+}$many $\beta \in Z$ such that $p_{\beta}$ is in the generic set. So for every $\gamma<\kappa^{+}$we can find $i_{\gamma}<\kappa^{+}$and $q_{\gamma} \geq p_{i_{\gamma}}$ such that $i_{\gamma} \in Z$, if $\gamma<\gamma^{\prime}$, then $i_{\gamma}<i_{\gamma^{\prime}}$ and for all $j \geq i_{\gamma+1}$, if $j \in Z$, then $q_{\gamma}$ forces that $p_{j}$ does not belong to the generic set. But then $\left\{q_{\gamma} \mid \gamma<\kappa^{+}\right\}$is an antichain, a contradiction.

5. Conclusion. Con $(Z F C)$ implies the consistency of $Z F C$ together with the following: There is an atomic Boolean algebra $B$ of power $\aleph_{1}$ such that $|A u t(B)|=\aleph_{\omega}$ (and if one wants $2^{\aleph_{0}}>\aleph_{\omega}$ ).

Proof. By Corollary 4 it is enough to show that Con(ZFC) implies the consistency of ZFC together with the following: $\left(2^{\aleph_{0}}>\aleph_{\omega}\right.$, if wanted, and $)$ there is a narrow $\omega_{1}$-tree $T$ with $\aleph_{\omega}$ branches. This is standard but let us sketch the proof: Assume CH. Let $P$ consist of four-tuples $p=\left(\alpha^{p}, T^{p}, u^{p},\left\{\eta_{i}^{p} \mid i \in u^{p}\right\}\right)$ such that $\alpha^{p}<\omega_{1}$, $T^{p}$ is a downwards closed countable subtree of $\omega \leq \alpha_{p}, u^{p}$ is a countable non-empty subset of $\aleph_{\omega}$ and for all $i \in u^{p}, \eta_{i}^{p}$ is a branch in $T^{p}$. The order is the obvious one: $p<q$ if $\alpha^{p}<\alpha^{p}, T^{q}\left\lceil\alpha^{p}+1=T^{p}\right.$, etc. Then $P$ is $\omega_{1}$-closed and by $\mathrm{CH}$, it has $\omega_{2}$-cc. $P$ also adds the required tree $T$. (There are no branches other than $\eta_{i}=\bigcup_{p \in G} \eta_{i}^{p}, i<\aleph^{\omega}$, because if $p$ forces that $b$ is a linearly ordered subtree of $T$ and for all $i<\aleph_{\omega}, b \neq \eta_{i}$, then by using the fact that $P$ is $\omega_{1}$-closed, we can find $q>p$ such that $q$ decides $b\left\lceil\alpha^{q}, b\left\lceil\alpha^{q} \neq \eta_{i}^{q}\left\lceil\alpha^{q}\right.\right.\right.$ for all $i \in u^{q}$ and no $t \in T^{q}$ is on top of $b\left\lceil\alpha^{q}\right.$. Then $q$ forces that the height of $b$ is $\leq \alpha^{q}$.) Now, if we want, we can add $>\aleph_{\omega}$ Cohen reals. As in the proof of Claim 4.1, we can see that this does not add branches to $T$.

\section{REFERENCES}

[Ro] J. Roitman, The number of automorphism of an atomic Boolean algebra, Pacific Journal of Mathematics, vol. 94 (1981), 231-242. MR 82j:03064

[Mo] J. D. Monk, Automorphism groups, J. D. Monk and R. Bonnet (ed.) Handbook of Boolean Algebras, vol. 2, North-Holland, Amsterdam, 1989, 517-546. CMP 21:10

Department of Mathematics, P.O. Box 4, 00014 University of Helsinki, Finland

E-mail address: thyttine@helsinki.fi

Institute of Mathematics, The Hebrew University, Jerusalem, Israel - and - Rutgers University, Department of Mathematics, New Brunswick, New Jersey

E-mail address: shelah@math.huji.ac.il 